\title{
PERSISTENCE IN DYNAMICAL SYSTEMS
}

\author{
BY \\ ZHI-DONG TENG AND KUI-CHEN DUAN \\ Xinjiang University, Urumqi, China
}

\begin{abstract}
In this paper, we study persistence and uniform persistence in dynamical systems. Necessary and sufficient conditions are given. These results are an extension of G. Butler, H. Freedman, and P. Waltman's discussions. Applying these results to two- and three-dimensional ecosystems, we obtain necessary and sufficient conditions about persistence and uniform persistence of these systems.
\end{abstract}

1. Introduction. Let $X$ be a locally compact metric space with metric $d, E$ be a nonempty closed subset of $X$. For any subset $S$ of $X$, boundary, closure, and interior of $S$ will be denoted by $b(S), \bar{S}$, and int $S$, respectively. Let $F$ be a dynamical system defined on the set $E$, that is $F=(E, R, \pi)$, where $R$ is the real numbers, $\pi: E \times R \rightarrow E$ is a continuous mapping such that $\pi(x, 0)=x$ and $\pi(\pi(x, t), s)=\pi(x, t+s)$ for all $x \in E$ and $t, s \in R$. In this paper, we assume throughout that $b(E)$ is an invariant set of the system $F$, i.e., $\pi(b(E) \times R) \subset b(E)$, and int $E \neq \varnothing$. For the $n$-species Kolmogorov system

$$
d x_{i} / d t=x_{i} f_{i}\left(x_{1}, x_{2}, \ldots, x_{n}\right), \quad i=1,2, \ldots, n,
$$

where the $f_{i}$ are continuous on $R_{+0}^{n}=\left\{\left(x_{1}, x_{2}, \ldots, x_{n}\right) \mid x_{i} \geq 0, i=1,2, \ldots, n\right\}$ and are such that the system satisfies existence, uniqueness, and continuability of solutions of initial value problems. $b\left(R_{+0}^{n}\right)$ is an invariant set of the system. Therefore the $n$-species Kolmogorov system is a special situation of the system $F$, i.e., $E=R_{+0}^{n}$. This shows it is important in mathematical ecology to study the system $F$ when $b(E)$ is an invariant set.

The concept of persistence plays an important role in mathematical ecology. Various definitions of persistence have been considered in articles [2, 3, 6, 8, 11]. Among these, persistence and uniform persistence seem to be the most suitable from the point of view of applications. In [2, 3], G. Butler, H. Freedman, and P. Waltman gave a method which studies persistence and uniform persistence of system $F$, and obtained a necessary and sufficient distinguishing criterion for uniform persistence of system $F$. But G. Butler et al. required that system $F$ has restrictive properties on the boundary $b(E)$. In particular, they required that system $F$ be dissipative and isolated on $b(E)$. Thus the range of applications subject to this criterion is 
restricted. In fact, these properties may be weakened considerably, and the property that system $F$ is dissipative on $b(E)$ may be abolished entirely. Therefore, in this paper, it is our purpose to give necessary and sufficient criteria of persistence and uniform persistence for system $F$ under weaker restrictions on the boundary $b(E)$. In the first place, we will give some new properties of limit sets of trajectories of system $F$. We will use these new properties to study persistence and uniform persistence of system $F$.

2. Main definitions and lemmas. For any $x \in E$, the trajectory, positive semitrajectory, and negative semi-trajectory of system $F$ through the point $x$ will be denoted by $r(x), r^{+}(x)$, and $r^{-}(x)$, respectively. The omega and alpha limit sets of the trajectory $r(x)$ will be denoted by $\Lambda^{+}(x), \Lambda^{-}(x)$, respectively.

Definition 1. Let $M \subset E$ be a nonempty set, if for any point $x \in M, \overline{r^{+}(x)}$ is compact, then we say that system $F$ is quasi-dissipative over $M$.

If system $F$ is quasi-dissipative over $M$, then for any point $x \in M, \Lambda^{+}(x)$ is a nonempty, compact, connected, and invariant set.

Definition 2. Let system $F$ be quasi-dissipative over $M \subset E$, then system $F$ is dissipative over $M$ if there is a compact set $N \subset E$ such that $\Lambda^{+}(x) \subset N$ for all $x \in M$.

If system $F$ is dissipative over $M$, then the set $\Omega=\bigcup_{x \in M} \Lambda^{+}(x)$ has compact closure $\bar{\Omega}$.

Definition 3. Let $M \subset E$ be a nonempty invariant set, then $M$ is an isolated invariant set if there is a neighborhood $U(M)$ of $M$ such that $M$ is a maximum invariant set in $U(M)$, in which case $U(M)$ is called an isolated neighborhood of $M$.

If $M$ is an isolated invariant set, then $M$ is a closed set. For any nonempty set $M \subset E$, the stable set and unstable set of $M$ are denoted by $S^{+}(M)=\left\{x \mid \Lambda^{+}(x) \neq\right.$ $\left.\varnothing, \Lambda^{+}(x) \subset M\right\}, S^{-}(M)=\left\{x \mid \Lambda^{-}(x) \neq \varnothing, \Lambda^{-}(x) \subset M\right\}$, respectively.

Definition 4. Let $M_{1}, M_{2}, \ldots, M_{k}$ be a pairwise disjoint sequence of nonempty sets, then $\left\{M_{1}, M_{2}, \ldots, M_{k}\right\}$ is called a cycle if there are points $Q_{1}, Q_{2}, \ldots, Q_{k}$ such that $Q_{i} \in S^{+}\left(M_{i}\right) \cap S^{-}\left(M_{i+1}\right), Q_{i} \notin M_{i} \cup M_{i+1}$ for $i=1,2, \ldots, k$, and $M_{k+1}=M_{1}$.

Definition 5. Let $A$ be a nonempty index set, $\left\{M_{\alpha} \mid \alpha \in A\right\}$ be a class of pairwise disjoint nonempty sets, then $\left\{M_{\alpha} \mid \alpha \in A\right\}$ is called acyclic if any finite subset of $\left\{M_{\alpha} \mid \alpha \in A\right\}$ does not form a cycle.

Let $B=\left\{x \mid x \in b(E), \overline{r^{+}(x)}\right.$ is compact $\}$ and $C=\bigcup_{x \in B} \Lambda^{+}(x)$, when $B \neq \varnothing$ then $C \neq \varnothing$.

Definition 6. Let $A$ be a nonempty index set, $\left\{M_{c} \mid \alpha \in A\right]$ be a class of pairwise disjoint nonempty sets, then $\left\{M_{\alpha} \mid \alpha \in A\right\}$ is called a covering of $C$ if all $M_{\alpha} \subset$ $b(E), M_{\alpha} \cap C \neq \varnothing$, and $C \subset \bigcup_{\alpha \in A} M_{\alpha}$.

The definitions that system $F$ is persistent and uniform persistent have been given in $[2,3]$. Here we only need to point out that if system $F$ is quasi-dissipative on the int $E$, then system $F$ is persistent if and only if for all $x \in$ int $E, \Lambda^{+}(x) \subset$ int $E$. Next we give several useful lemmas. They represent some new properties of limit 
sets of trajectories of system $F$. In the first place, we need the following hypothesis.

$\mathrm{H}$. There is a covering $\left\{M_{\alpha} \mid \alpha \in A\right\}$ of $C$ such that

a) all $M_{\alpha}$ are isolated invariant set of system $F$,

b) $\left\{M_{\alpha} \mid \alpha \in A\right\}$ is acyclic,

c) for any compact set $M \subset E$, there is only a finite subset $\left\{M_{1}, M_{2}, \ldots, M_{k}\right\}$ of $\left\{M_{\alpha} \mid \alpha \in A\right\}$ such that $M \cap M_{i} \neq \varnothing$ for $i=1,2, \ldots, k$.

Lemma 1. Let $M \subset E$ be an isolated invariant set of system $F, x \in E$ and $\overline{r^{+}(x)}$ be compact, $\Lambda^{+}(x) \cap M \neq \varnothing$. Then $\Lambda^{+}(x) \subset M$ or $\Lambda^{+}(x) \cap\left(S^{+}(M)-M\right) \neq \varnothing$ and $\Lambda^{+}(x) \cap\left(S^{-}(M)-M\right) \neq \varnothing$.

Proof. Let $U(M)$ be an isolated neighborhood of $M$. Suppose $\Lambda^{+}(x)-M \neq \varnothing$. Since $\Lambda^{+}(x) \cap M$ is nonempty and compact, we can select a neighborhood $N$ of $\Lambda^{+}(x) \cap M$ such that $\bar{N} \subset U(M), \Lambda^{+}(x) \cap b(N) \neq \varnothing$, and $\bar{N}$ is compact. Because of the property of $\Lambda^{+}(x)$, we can choose two time sequences $\left\{t_{k}\right\},\left\{s_{k}\right\}, t_{k}>0, s_{k}<0$ such that $t_{k} \rightarrow+\infty, x_{k}=\pi\left(x, t_{k}\right) \rightarrow \Lambda^{+}(x) \cap M$ as $k \rightarrow \infty$ and $\pi\left(x_{k},\left(s_{k} 0\right)\right) \subset N$, $\pi\left(x_{k}, s_{k}\right) \in b(N)$ for $k=1,2, \ldots$.

Let $y_{k}=\pi\left(x, t_{k}+s_{k}\right)=\pi\left(x_{k}, s_{k}\right)$. Since $b(N)$ is compact, we can assume $y_{k} \rightarrow y_{0}$ as $k \rightarrow \infty, y_{0} \in b(N)$, and $y_{0} \notin \Lambda^{+}(x) \cap M$. Similar to the method of proof of Theorem 4.1 in [3], we can prove $y_{0} \in S^{+}(M)$ and $y_{0} \in \Lambda^{+}(x)$. Thus we obtain $y_{0} \in \Lambda^{+}(x) \cap\left(S^{+}(M)-M\right)$, and therefore $\Lambda^{+}(x) \cap\left(S^{+}(M)-M\right) \neq \varnothing$.

Similarly, we can prove $\Lambda^{+}(x) \cap\left(S^{-}(M)-M\right) \neq \varnothing$. This completes the proof.

Lemma 2. Let $M \subset E$ be an isolated invariant set of system $F, x \in E, \overline{r^{-}(x)}$ is compact and $\Lambda^{-}(x) \cap M \neq \varnothing$. Then $\Lambda^{-}(x) \subset M$ or $\Lambda^{-}(x) \cap\left(S^{+}(M)-M\right) \neq \varnothing$ and $\Lambda^{-}(x) \cap\left(S^{-}(M)-M\right) \neq \varnothing$.

The proof of Lemma 2 is similar to Lemma 1, and so we omit it.

Lemma 3. Suppose that hypothesis $\mathrm{H}$ holds, $x \in E, \overline{r^{+}(x)}$ is compact and $\Lambda^{+}(x) \subset$ $b(E)$. Then there is a $M_{\alpha} \in\left\{M_{\alpha} \mid \alpha \in A\right\}$ such that $\Lambda^{+}(x) \subset M_{\alpha}$.

Proof. Since $\Lambda^{+}(x)$ is compact, by the hypothesis $\mathrm{H}$ there is only a finite subset $\left\{M_{1}, M_{2}, \ldots, M_{k}\right\}$ of $\left\{M_{\alpha} \mid \alpha \in A\right\}$ such that $\Lambda^{+}(x) \cap M_{i} \neq \varnothing$ for $i=1,2, \ldots, k$. Therefore $\Lambda^{+}(x) \cap\left(\bigcup_{i=1}^{k} M_{i}\right) \neq \varnothing$ and $\Lambda^{+}(x) \cap\left(\bigcup_{\alpha \in A} M_{\alpha}-\bigcup_{i=1}^{k} M_{i}\right)=\varnothing$. Suppose $\Lambda^{+}(x)-M_{\alpha} \neq \varnothing$ for any $\alpha \in A$. Then there is $M_{i 1} \in\left\{M_{1}, M_{2}, \ldots, M_{k}\right\}$ such that $M_{i 1} \cap \Lambda^{+}(x) \neq \varnothing$ and $\Lambda^{+}(x)-M_{i 1} \neq \varnothing$. By Lemma 1 we obtain $\Lambda^{+}(x) \cap$ $\left(S^{-}\left(M_{i 1}\right)-M_{i 1}\right) \neq \varnothing$. Choose $p_{1} \in \Lambda^{+}(x) \cap\left(S^{-}\left(M_{i 1}\right)-M_{i 1}\right)$. Then $p_{1} \in B$ and $\Lambda^{+}\left(p_{1}\right) \subset \bigcup_{\alpha \in A} M_{\alpha}$, since $\Lambda^{+}\left(p_{1}\right)$ is a connective set and all $M_{\alpha}$ are pairwise disjoint sets. Therefore there is a $M_{i 2} \in\left\{M_{1}, M_{2}, \ldots, M_{k}\right\}$ such that $\Lambda^{+}\left(p_{1}\right) \subset M_{i 2}$. If $p_{1} \in M_{i 2}$, then $\Lambda^{-}\left(p_{1}\right) \subset M_{i 2}$, since $\Lambda^{-}\left(p_{1}\right) \subset M_{i 1}$. Hence we must have $M_{i 1}=M_{i 2}$ and $p_{1} \in M_{i 1}$. But this contradicts with $p_{1} \in S^{-}\left(M_{i 1}\right)-M_{i 1}$. Therefore this shows that $p_{1} \in S^{+}\left(M_{i 2}\right) \cap S^{-}\left(M_{i 1}\right)$ and $p_{1} \notin M_{i 1} \cup M_{i 2}$.

Since $\Lambda^{+}(x) \cap M_{i 2} \neq \varnothing$ and $\Lambda^{+}(x)-M_{i 2} \neq \varnothing$, therefore we can repeat the preceding arguments and obtain that there are $p_{2} \in \Lambda^{+}(x)$ and $M_{i 3} \in\left\{M_{1}, M_{2}, \ldots, M_{k}\right\}$ such that $p_{2} \in S^{+}\left(M_{i 3}\right) \cap S^{-}\left(M_{i 2}\right)$ and $p_{2} \neq M_{i 2} \cup M_{i 3}$. Continuing with these 
arguments, since $M_{1}, M_{2}, \ldots, M_{k}$ are finite, we can obtain a cycle $\left\{M_{i 1}, M_{i 2}, \ldots\right.$, $\left.M_{i m}\right\} \subset\left\{M_{1}, M_{2}, \ldots, M_{k}\right\}$. But this contradicts hypothesis $\mathrm{H}$. Hence there is a $M_{\alpha} \in\left\{M_{\alpha} \mid \alpha \in A\right\}$ such that $\Lambda^{+}(x) \subset M_{\alpha}$, completing the proof.

Similarly, we have the following lemma.

LEMma 4. Suppose that hypothesis H holds, $x \in E, \overline{r^{-}(x)}$ is compact and $\Lambda^{-}(x) \subset$ $b(E)$. Then there is a $M_{c} \in\left\{M_{\alpha} \mid \alpha \in A\right\}$ such that $\Lambda^{-}(x) \subset M_{c}$.

3. Main theorems. In [2,3], G. Butler et al. required that $\left\{M_{\alpha} \mid \alpha \in A\right\}$ is a finite set, all $M_{\alpha}$ are compactly isolated invariant sets, system $F$ is dissipative on the set $E$ and $B=b(E)$. Here we will weaken their conditions. We allow that $\left\{M_{a} \mid \alpha \in A\right\}$ may be an infinite set, all $M_{\alpha}$ may be noncompactly isolated invariant sets, system $F$ is only quasi-dissipative or dissipative on int $E$, and $B$ may not equal $b(E)$. We have the following results.

Theorem 1. Suppose that system $F$ is quasi-dissipative on int $E$, hypothesis $\mathrm{H}$ holds and $B \neq \varnothing$. Then system $F$ is persistent if and only if $S^{+}\left(M_{c}\right) \cap$ int $E=\varnothing$ for any $M_{\alpha} \in\left\{M_{\alpha} \mid \alpha \in A\right\}$.

Proof. As it is easy to prove the necessary condition, we prove only the sufficient condition. If system $F$ is not persistent, then there is a $x \in \operatorname{int} E$ such that $\Lambda^{+}(x) \cap$ $b(E) \neq \varnothing$. Since $\Lambda^{+}(x) \cap b(E)$ is a compact invariant set, then $\Lambda^{+}(x) \cap C \neq \varnothing$. By hypothesis $\mathrm{H}$ we obtain that there is a finite subset $\left\{M_{1}, M_{2}, \ldots, M_{k}\right\}$ of $\left\{M_{\alpha} \mid \alpha \in\right.$ $A\}$ such that $\Lambda^{+}(x) \cap M_{i} \neq \varnothing$, and therefore that $\Lambda^{+}(x) \cap\left(\bigcup_{i=1}^{k} M_{i}\right) \neq \varnothing$ and $\Lambda^{+}(x) \cap\left(\bigcup_{c \in A} M_{\alpha}-\bigcup_{i=1}^{k} M_{i}\right)=\varnothing$. Since $S^{+}\left(M_{i}\right) \cap$ int $E=\varnothing$, then $\Lambda^{+}(x)-M_{i} \neq \varnothing$ for $i=1,2, \ldots, k$. Choose a $M_{i 1} \in\left\{M_{1}, M_{2}, \ldots, M_{k}\right\}$. By Lemma 1 we obtain $\Lambda^{+}(x) \cap\left(S^{+}\left(M_{i 1}\right)-M_{i 1}\right) \neq \varnothing$. Choose $p_{1} \in \Lambda^{+}(x) \cap\left(S^{+}\left(M_{i 1}\right)-M_{i 1}\right)$. Then $p_{1} \in b(E) \cap B$ and $\Lambda^{-}\left(p_{1}\right) \subset \Lambda^{+}(x) \cap b(E)$. By Lemma 4 we obtain that there is a $M_{i 2} \in\left\{M_{1}, M_{2}, \ldots, M_{k}\right\}$ such that $\Lambda^{-}\left(p_{1}\right) \subset M_{i 2}$. According to the proof of Lemma 3, we have $p_{1} \notin M_{i 2}$. This shows that $p_{1} \in S^{+}\left(M_{i 1}\right) \cap S^{-}\left(M_{i 2}\right)$ and $p_{1} \notin M_{i 1} \cup M_{i 2}$.

For $M_{i 2}$, we can repeat the preceding arguments and obtain that there are $p_{2} \in$ $\Lambda^{+}(x)$ and $M_{i 3} \in\left\{M_{1}, M_{2}, \ldots, M_{k}\right\}$ such that $p_{2} \in S^{+}\left(M_{i 2}\right) \cap S^{-}\left(M_{i 3}\right)$ and $p_{2} \notin$ $M_{i 2} \cup M_{i 3}$. Continuing with these arguments, since $\left\{M_{1}, M_{2}, \ldots, M_{k}\right\}$ is a finite set, we can finally obtain a cycle $\left\{M_{i 1}, M_{i 2}, \ldots, M_{i m}\right\} \subset\left\{M_{1}, M_{2}, \ldots, M_{k}\right\}$. But this contradicts hypothesis $\mathrm{H}$, showing that system $F$ must be persistent. This completes the proof.

ThEOREM 2. Suppose that system $F$ is quasi-dissipative on int $E$ and $B=\varnothing$. Then system $F$ must be persistent.

The proof of this theorem is easy, and so we omit it.

THEOREM 3. Suppose that system $F$ is dissipative on int $E$, hypothesis $\mathrm{H}$ holds and $B \neq \varnothing$. Then system $F$ is uniform persistent if and only if $S^{+}\left(M_{n}\right) \cap$ int $E=\varnothing$ for any $M_{\alpha} \in\left\{M_{\alpha} \mid \alpha \in A\right\}$.

Proof. The proof of the necessary condition is easy, and so we prove only the sufficient condition. In the first place, the conditions of Theorem 1 hold when the 
conditions of Theorem 3 hold, and therefore system $F$ is persistent. Let $\Omega=$ $\bigcup_{x \in \operatorname{int} E} \Lambda^{+}(x)$. Then $\Omega \subset$ int $E, \bar{\Omega}$ is compact and $\Omega, \bar{\Omega}$ are both invariant sets. By hypothesis $\mathrm{H}$, we obtain that there is only a finite subset $\left\{M_{1}, M_{2}, \ldots, M_{k}\right\}$ of $\left\{M_{\alpha} \mid \alpha \in A\right\}$ such that $\Omega \cap M_{i} \neq \varnothing$. Thus $\bar{\Omega} \cap \bigcup_{i=1}^{k} M_{i} \neq \varnothing$ and $\bar{\Omega} \cap\left(\bigcup_{\alpha \in A} M_{\alpha}-\right.$ $\left.\bigcup_{i=1}^{k} M_{i}\right)=\varnothing$.

Consider $M_{1} \cap \bar{\Omega}, M_{2} \cap \bar{\Omega}, \ldots, M_{k} \cap \bar{\Omega}$. Then they are pairwise disjoint nonempty invariant sets. Let $U\left(M_{\alpha}\right)$ be an isolated neighborhood of $M_{\alpha}$. We can choose two compact neighborhoods $U_{i}, V_{i}$ of $M_{i} \cap \bar{\Omega}$ such that $U_{i} \subset$ int $V_{i}$, $M_{i} \cap \bar{\Omega} \subset U_{i}, V_{i} \subset U\left(M_{i}\right)$ for $i=1,2, \ldots, k$ and $V_{1}, V_{2}, \ldots, V_{k}$ are pairwise disjoint.

Suppose that system $F$ is not uniformly persistent. Then according to a similar method of proof of the main theorem in [2], we can obtain that there are $\omega_{n}=$ $r\left(p_{n}\right) \subset \Omega, p_{n} \in$ int $E$, and a nonempty, compact, and invariant set $\omega \subset \bar{\Omega}$ such that $\rho\left(\omega_{n}, \omega\right) \rightarrow 0$ as $n \rightarrow \infty$ and $b^{\prime}=\omega \cap C \neq \varnothing, b^{\prime} \subset \bigcup_{i=1}^{k} M_{i} \cap \bar{\Omega}$, where $\rho$ is the Hausdorff metric on $K$, where $K=\{A \mid A \subset \bar{\Omega}$ is nonempty and compact subset $\}$. Let $b_{1}=b^{\prime} \cap M_{i 1} \neq \varnothing$ and $M_{i 1} \in\left\{M_{1}, M_{2}, \ldots, M_{k}\right\}$. Then there is a $p_{1} \in b\left(U_{i 1}\right) \cap b(E)$ such that $\Lambda^{+}\left(p_{1}\right) \subset M_{i 1} \cap \bar{\Omega}, \Lambda^{-}\left(p_{1}\right) \subset \omega$, and $p_{1} \in$ $\bar{\Omega}$. By Lemma 4 , we obtain that there is $M_{i 2} \in\left\{M_{1}, M_{2}, \ldots, M_{k}\right\}$ such that $\Lambda^{-}\left(p_{1}\right) \subset M_{i 2}$. Therefore $p_{1} \in S^{+}\left(M_{i 1}\right) \cap S^{-}\left(M_{i 2}\right)$ and $p_{1} \notin M_{i 1} \cup M_{i 2}$. Since $b_{2}=$ $b^{\prime} \cap M_{i 2} \neq \varnothing$, we can repeat the preceding arguments and obtain that there are $p_{2} \in$ $b\left(U_{i 2}\right) \cap b(E)$ and $M_{i 3} \in\left\{M_{1}, M_{2}, \ldots, M_{k}\right\}$ such that $p_{2} \in S^{+}\left(M_{i 2}\right) \cap S^{-}\left(M_{i 3}\right)$ and $p_{2} \notin M_{i 2} \cup M_{i 3}$. Continuing with these arguments, since $\left\{M_{1}, M_{2}, \ldots, M_{k}\right\}$ is a finite set, we can finally obtain a cycle $\left\{M_{i 1}, M_{i 2}, \ldots, M_{i m}\right\} \subset\left\{M_{1}, M_{2}, \ldots, M_{k}\right\}$. But this contradicts hypothesis $\mathrm{H}$, and shows that system $F$ must be uniformly persistent, completing the proof.

Theorem 4. Suppose that system $F$ is dissipative on int $E$ and $B=\varnothing$. Then system $F$ must be uniformly persistent.

The proof of this theorem is obvious.

4. Applications. In this section, the above theorems will be applied to obtain criteria of persistence and uniform persistence for the general two-species and threespecies Kolmogorov systems. These systems may be predator-prey, competitive, cooperative, etc. The work in applying the above theorems then is to check the isolated invariant and acyclic conditions, i.e., the conditions a) and b) of hypothesis $\mathrm{H}$. This question will be obviously represented in the following discussions of this section. In the first place, we have set $E=R_{+0}^{2}$ and $R_{+0}^{3}$ (see Sec. 1), and the condition that system $F$ is quasi-dissipative or dissipative on int $E$ then becomes that all solutions with positive initial conditions are bounded or ultimately bounded in future time.

Consider the general two-species system

$$
\begin{aligned}
& d x_{1} / d t=x_{1} f_{1}\left(x_{1}, x_{2}\right), \\
& d x_{2} / d t=x_{2} f_{2}\left(x_{1}, x_{2}\right) .
\end{aligned}
$$

Suppose that the functions $f_{1}, f_{2}$ are continuous on $R_{+0}^{2}$ and are such that system 
(1) satisfies existence, uniqueness, and continuability of solutions of initial value problems. Let set $A$ represent all equilibrium points located within $R_{0}^{2}=b\left(R_{+0}^{2}\right)$. $R_{0}^{2}$ is an invariant set of system (1). Let $R_{+}^{2}=$ int $R_{+0}^{2}$. We have the following theorem.

THEOREM 5. Suppose that all solutions of system (1) with positive initial conditions are bounded (ultimately bounded) in future time and there is a neighborhood $U(A)$ of $A$ such that system (1) has no equilibrium in $U(A) \cap R_{+}^{2}$. Then system (1) is persistent (uniformly persistent) if and only if $S^{+}(A) \cap R_{+}^{2}=\varnothing$.

Proof. We give only the proof of the sufficient condition. In the first place, since $S^{+}(A) \cap R_{+}^{2}=\varnothing$ we obtain $S^{+}\left(R_{0}^{2}\right) \cap R_{+}^{2}=\varnothing$.

For any $x \in R_{0}^{2}$, if $x$ is not an equilibrium, by the continuity of the $f_{1}$ and $f_{2}$, there is a neighborhood $U(x)$ such that system (1) has no equilibrium in $U(x) \cap R_{+}^{2}$. If $x$ is an equilibrium then there is a neighborhood $U(x) \subset U(A)$ such that system (1) has no equilibrium in $U(x) \cap R_{+}^{2}$. Let $U\left(R_{0}^{2}\right)=\bigcup_{x \in R_{0}^{2}} U(x)$. Then $U\left(R_{0}^{2}\right)$ is a neighborhood of $R_{0}^{2}$. System (1) has no equilibrium in $U\left(R_{0}^{2}\right) \cap R_{+}^{2}$. For any $x \in U\left(R_{0}^{2}\right) \cap R_{+}^{2}$, consider the positive semi-trajectory $r^{+}(x)$ and omega limit set $\Lambda^{+}(x)$. Since $S^{+}\left(R_{0}^{2}\right) \cap R_{+}^{2}=\varnothing$, then $\Lambda^{+}(x) \cap R_{+}^{2} \neq \varnothing$. If there is no equilibrium in $\Lambda^{+}(x)$, then $\Lambda^{+}(x)$ must be a closed trajectory. Since $R_{0}^{2}$ is an invariant set, it follows that $\Lambda^{+}(x) \subset R_{+}^{2}$. Thus system (1) has an equilibrium $p$ in the domain of which the boundary is $\Lambda^{+}(x)$ and $p \in R_{+}^{2}$. Therefore $p \notin U\left(R_{0}^{2}\right) \cap R_{+}^{2}$. This shows $\Lambda^{+}(x)-U\left(R_{0}^{2}\right) \neq \varnothing$. If there is an equilibrium in $\Lambda^{+}(x)$ and if all equilibrium in $\Lambda^{+}(x)$ is located in $R_{0}^{2}$, by the properties of two-dimensional systems, for $y \notin$ $\Lambda^{+}(x) \cap R_{+}^{2}$ we have $\Lambda^{+}(y) \subset A$, which contradicts $S^{+}(A) \cap R_{+}^{2}=\varnothing$. Therefore there is an equilibrium $p \in \Lambda^{+}(x)$ such that $p \in R_{+}^{2}$ and $p \notin U\left(R_{0}^{2}\right) \cap R_{+}^{2}$. This shows that $\Lambda^{+}(x)-U\left(R_{0}^{2}\right) \neq \varnothing$. Therefore under all possible circumstances $r^{+}(x)-U\left(R_{0}^{2}\right) \neq \varnothing$. This shows that $R_{0}^{2}$ is a maximum invariant set in $U\left(R_{0}^{2}\right)$ and $R_{0}^{2}$ must be an isolated invariant set.

Since $S^{+}\left(R_{0}^{2}\right) \cap R_{+}^{2}=\varnothing, R_{0}^{2}$ does not form a cycle. Thus by the above theorems, we obtain that system (1) is persistent (uniformly persistent). This completes the proof.

The following corollary is convenient for applications.

COROLlaRY 1. Let all solutions of system (1) with positive initial conditions be bounded (ultimatley bounded) in future time. If there are equilibria $\left(x_{1}^{*}, 0\right)$ and $\left(0, x_{2}^{*}\right)$ where $x_{1}^{*}>0, x_{2}^{*}>0$, then assume that $f_{2}\left(x_{1}^{*}, 0\right)>0$ and $f_{1}\left(0, x_{2}^{*}\right)>0$, $\max \left\{f_{1}(0,0), f_{2}(0,0\}>0\right.$. Then system (1) is persistent (uniformly persistent).

The proof of the corollary is clear and so we omit it.

Next we consider the general three-species system

$$
\begin{aligned}
& d x_{1} / d t=x_{1} f_{1}\left(x_{1}, x_{2}, x_{3}\right), \\
& d x_{2} / d t=x_{2} f_{2}\left(x_{1}, x_{2}, x_{3}\right), \\
& d x_{3} / d t=x_{3} f_{3}\left(x_{1}, x_{2}, x_{3}\right) .
\end{aligned}
$$


Suppose that the functions $f_{i}$ are continuous on $R_{+0}^{3}$ such that system (2) satisfies existence, uniqueness, and continuability of solutions of initial value problems. Let $x=\left(x_{1}, x_{2}, x_{3}\right), R_{+0}^{2}\left(x_{i}, x_{j}\right)=\left\{x \mid x_{i} \geq 0, x_{j} \geq 0, x_{k}=0\right\}$ and $R_{+}^{2}\left(x_{i}, x_{j}\right)=$ $\left\{x \mid x_{i}>0, x_{j}>0, x_{k}=0\right\}, R_{+}^{1}\left(x_{i}\right)=\left\{x \mid x_{i}>0, x_{j}=0, x_{k}=0\right\}, R_{+}^{3}=\operatorname{int} R_{+0}^{3}$, $R_{0}^{3}=b\left(R_{+0}^{3}\right) . R_{0}^{3}, R_{+0}^{2}\left(x_{i}, x_{j}\right), R_{+}^{1}\left(x_{i}\right)$, etc., are all invariant sets of system (2). Let the set $A$ represent all equilibria located within $R_{0}^{3}$. We give the following decomposition of the set $A$,

$$
A=\bigcup_{m} C_{m},
$$

where every $C_{m}$ is a connected component of set $A$. Set $\left\{C_{m}\right\}$ may be an infinite set. We need the following hypotheses:

$\mathrm{H}_{1}$. All $C_{m}$ are isolated invariant sets of system (2).

$\mathrm{H}_{2} .\left\{C_{m}\right\}$ is acyclic.

$\mathrm{H}_{3}$. there are no closed trajectories of system (2) in each $R_{+}^{2}\left(x_{i}, x_{j}\right)$.

Lemma 5. Let the hypothesis $\mathrm{H}_{1}$ hold. Then for any $R>0$, there is only a finite subset $\left\{C_{1}, C_{2}, \ldots, C_{k}\right\}$ of $\left\{C_{m}\right\}$ such that $S_{R} \cap C_{i} \neq \varnothing$, where $S_{R}=\{x|| x \mid \leq R\}$.

Proof. If there are infinitely many $C_{m}$ satisfying $S_{R} \cap C_{m} \neq \varnothing$, choose $p_{m} \in$ $S_{R} \cap C_{m}$. Then $\left\{p_{m}\right\}$ is a bounded infinite set of points. Therefore there is a sequence $\left\{p_{m k}\right\} \subset\left\{p_{m}\right\}$ such that $p_{m k} \rightarrow p$ as $k \rightarrow \infty, p_{m k} \neq p$ for $k=1,2, \ldots$ and $p \in A \cap S_{R}$. Thus there is a $C_{m^{\prime}} \in\left\{C_{m}\right\}$ such that $p \in C_{m^{\prime}} \cap S_{R}$. Let $U\left(C_{m^{\prime}}\right)$ be an isolated neighborhood of $C_{m^{\prime}}$. If $C_{m} \cap U\left(C_{m^{\prime}}\right) \neq \varnothing$, then $C_{m^{\prime}} \cup\left(C_{m} \cap U\left(C_{m^{\prime}}\right)\right)$ is an invariant set in $U\left(C_{m^{\prime}}\right)$. Hence $C_{m} \cap U\left(C_{m^{\prime}}\right)=\varnothing$ for $m \neq m^{\prime}$. But there is a $K$ such that $p_{m k} \in U\left(C_{m^{\prime}}\right)$ for $k>K$. Hence $C_{m k} \cap U\left(C_{m^{\prime}}\right) \neq \varnothing$ for $k>K$. This contradiction completes the proof.

Lemma 6. Let hypotheses $\mathrm{H}_{1}-\mathrm{H}_{3}$ hold, $x \in R_{0}^{3}$, and $r^{+}(x)$ is bounded. Then $\Lambda^{+}(x) \subset A$.

Proof. There is a $R>0$ such that $\Lambda^{+}(x) \subset S_{R}$. By Lemma 5 there is a finite subset $\left\{C_{1}, C_{2}, \ldots, C_{k}\right\} \subset\left\{C_{m}\right\}$ such that $\Lambda^{+}(x) \subset \bigcup_{i=1}^{k} C_{i} \neq \varnothing$ and $\Lambda^{+}(x) \cap\left(\bigcup_{m} C_{m}-\bigcup_{i=1}^{k} C_{i}\right)=\varnothing$. Let $\Lambda^{+}(x) \subset R_{+0}^{2}\left(x_{i}, x_{j}\right)$. By hypothesis $\mathrm{H}_{3}$ we obtain that there is an equilibrium in $\Lambda^{+}(x)$, and so $\Lambda^{+}(x) \cap A \neq \varnothing$. If $\Lambda^{+}(x)-A \neq$ $\varnothing$, then there is a $C_{i 1} \subset\left\{C_{1}, C_{2}, \ldots, C_{k}\right\}$ such that $\Lambda^{+}(x) \cap C_{i 1} \neq \varnothing$ and $\Lambda^{+}(x)-C_{i 1} \neq \varnothing$. By Lemma 1 we can choose a point $q \in \Lambda^{+}(x) \cap\left(S^{+}\left(C_{i 1}\right)-C_{i 1}\right)$ where $q$ is not an equilibrium. By the properties of a planar dynamical system, there is a $C_{i 2} \in\left\{C_{1}, C_{2}, \ldots, C_{k}\right\}$ such that $\Lambda^{-}(q) \subset C_{i 2}$. Hence $q \in S^{+}\left(C_{i 1}\right) \cap$ $S^{-}\left(C_{i 2}\right)$ and $q \notin C_{i 1} \cup C_{i 2}$. Similarly to the proof of Lemma 3, we obtain a cycle $\left\{C_{i 1}, C_{i 2}, \ldots, C_{i m}\right\} \subset\left\{C_{1}, C_{2}, \ldots, C_{k}\right\}$, contradicting hypothesis $\mathrm{H}_{2}$. Hence $\Lambda^{+}(x) \subset A$, completing the proof.

Theorem 6. Let hypotheses $\mathrm{H}_{1}-\mathrm{H}_{3}$ hold, and all solutions of system (2) with positive initial conditions be bounded (ultimately bounded) in future time. Then system (2) is persistent (uniformly persistent) if and only if $S^{+}\left(C_{m}\right) \cap R_{+}^{3}=\varnothing$ for every $C_{m} \in\left\{C_{m}\right\}$. 
Proof. Since $A \neq \varnothing$, then $B=\left\{x \mid x \in R_{0}^{3}, r^{+}(x)\right.$ is bounded $\} \neq \varnothing$. By Lemma 6, we have that $A=\bigcup_{x \in B} \Lambda^{+}(x)$. We choose $\left\{C_{m}\right\}$ as a covering of $A$. By Lemma 5 and hypotheses $\mathrm{H}_{1}-\mathrm{H}_{3}$ we obtain that hypothesis $\mathrm{H}$ holds. Thus from Theorem 1 and Theorem 3 we get that this theorem holds, completing the proof.

In this theorem, the boundedness of solutions shows that, although the population may grow to infinity in the absence of predation and/or competition, every population only grows to finiteness when all populations exist. This condition can often be eliminated by constructing Lyapunov functions. The hypothesis $\mathrm{H}_{1}$ shows that, if three populations near enough to the equilibrium station $C_{m}$ (i.e., distance $d\left(\left(x_{1}, x_{2}, x_{3}\right), C_{m}\right)$ is small enough) at some time $t_{0}$, then either they tend to $C_{m}$ or they tend away from $C_{m}$ as $t \rightarrow+\infty$. If every $C_{m}$ is an equilibrium, then hypothesis $\mathrm{H}_{1}$ can be checked by computing eigenvalues of the linearization about every equilibrium. The check of hypothesis $\mathrm{H}_{2}$ depends on the type of populations being modeled (predator-prey, competitive, cooperative, etc.). One interpretation of the work in $[3,6,7]$ is that the principal effort was equivalent to verifying the acyclic condition. The hypothesis $\mathrm{H}_{2}$ shows that, in the absence of one of the three populations, the other two populations cannot occur in a periodic situation. This hypothesis can often be eliminated by the Dulac Criterion.

Let sets $A_{i}$ and $B_{k}$ represent all equilibria located in $R_{+}^{1}\left(x_{i}\right)$ and $R_{+}^{2}\left(x_{i}, x_{j}\right)$, respectively. We give the following decompositions of $A_{i}$ and $B_{k}$.

$$
A_{i}=\bigcup_{m} A_{i m}, \quad B_{k}=\bigcup_{m} B_{k m},
$$

where every $A_{i m}, B_{k m}$ is a connected component of $A_{i}, B_{k}$. We need the following hypotheses:

$\mathrm{H}_{4}$. All $A_{i m}, B_{k m}$ are isolated invariant sets of system (2).

$\mathrm{H}_{5}$. Every $\left\{B_{k m}\right\}$ is acyclic.

$\mathrm{H}_{6}$. All solutions with positive intial conditions are bounded (ultimately bounded) in future time.

$\mathrm{H}_{7}$. For every $A_{i}, S^{+}\left(A_{i}\right) \cap R_{+}^{2}\left(x_{i}, x_{j}\right)=\varnothing$ or $S^{-}\left(A_{i}\right) \cap R_{+}^{2}\left(x_{i}, x_{j}\right)=\varnothing$.

$\mathrm{H}_{8}$. For every $A_{i m}, B_{k m}, S^{+}\left(A_{i m}\right) \cap R_{+}^{3}=\varnothing, S^{+}\left(B_{k m}\right) \cap R_{+}^{3}=\varnothing$.

$\mathrm{H}_{9} . f_{1}(0,0,0)>0, f_{2}(0,0,0)<0, f_{3}(0,0,0)<0$.

$\mathrm{H}_{10} . f_{1}(0,0,0)>0, f_{2}(0,0,0)>0, f_{3}(0,0,0)<0$.

$\mathrm{H}_{11} \cdot f_{1}(0,0,0)>0, f_{2}(0,0,0)>0, f_{3}(0,0,0)>0$.

We have the following theorems, which are convenient for applications.

THEOREM 7. Let hypotheses $\mathrm{H}_{3}-\mathrm{H}_{9}$ and one of the following conditions hold:

a) $A_{1}=\varnothing$,

b) $A_{2}=A_{3}=\varnothing$;

c) for $i=2, j=3$, or $i=3, j=2, A_{i}=\varnothing$, and $A_{j} \neq \varnothing, S^{+}\left(A_{j}\right) \cap$ $R_{+}^{2}\left(x_{1}, x_{j}\right)=\varnothing$ or $S^{-}\left(A_{1}\right) \cap R_{+}^{2}\left(x_{1}, x_{j}\right)=\varnothing ;$ 
d) $A_{1}, A_{2}$, and $A_{3}$ are nonempty, for $i=2,3, S^{+}\left(A_{i}\right) \cap R_{+}^{2}\left(x_{1}, x_{i}\right)=\varnothing$;

e) $A_{1}, A_{2}$, and $A_{3}$ are nonempty, for $i=2, j=3$ or $i=3, j=2$, $S^{+}\left(A_{i}\right) \cap R_{+}^{2}\left(x_{1}, x_{i}\right)=\varnothing$ and $S^{-}\left(A_{1}\right) \cap R_{+}^{2}\left(x_{1}, x_{j}\right)=\varnothing$.

Then system (2) is persistent (uniformly persistent).

We can obtain a theorem which is similar to Theorem 7 under the hypotheses $\mathrm{H}_{3}-\mathrm{H}_{8}$ and $\mathrm{H}_{10}$ holding.

THEOREM 8. Let hypotheses $\mathrm{H}_{3}-\mathrm{H}_{9}, \mathrm{H}_{11}$ and one of the following conditions hold:

a) one of $A_{1}, A_{2}, A_{3}$ is empty set;

b) there are $i$ and $j$ such that $S^{+}\left(A_{i}\right) \cap R_{+}^{2}\left(x_{i}, x_{j}\right)=\varnothing$ and $S^{-}\left(A_{i}\right) \cap R_{+}^{2}\left(x_{i}, x_{j}\right)$ $=\varnothing$

c) if $S^{+}\left(A_{i}\right) \cap R_{+}^{2}\left(x_{i}, x_{j}\right) \neq \varnothing$, then one of the following conditions holds: (1) $S^{-}\left(A_{i}\right) \cap R_{+}^{2}\left(x_{i}, x_{k}\right)=\varnothing ;(2) S^{+}\left(A_{k}\right) \cap R_{+}^{2}\left(x_{i}, x_{k}\right)=\varnothing ;$ (3) $S^{-}\left(A_{k}\right) \cap$ $R_{+}^{2}\left(x_{k}, x_{j}\right)=\varnothing ;(4) S^{+}\left(A_{j}\right) \cap R_{+}^{2}\left(x_{j}, x_{k}\right)=\varnothing ;(5) S^{-}\left(A_{j}\right) \cap R_{+}^{2}\left(x_{j}, x_{i}\right)=\varnothing ;$

d) if $S^{-}\left(A_{i}\right) \cap R_{+}^{2}\left(x_{i}, x_{j}\right) \neq \varnothing$, then one of the following conditions holds: (1) $S^{+}\left(A_{i}\right) \cap R_{+}^{2}\left(x_{i}, x_{k}\right)=\varnothing ;(2) S^{-}\left(A_{k}\right) \cap R_{+}^{2}\left(x_{i}, x_{k}\right)=\varnothing ;$ (3) $S^{+}\left(A_{j}\right) \cap$ $R_{+}^{2}\left(x_{j}, x_{i}\right)=\varnothing ;(4) S^{-}\left(A_{j}\right) \cap R_{+}^{2}\left(x_{j}, x_{k}\right)=\varnothing ;(5) S^{+}\left(A_{k}\right) \cap R_{+}^{2}\left(x_{k}, x_{j}\right)=\varnothing$.

Then system (2) is persistent (uniformly persistent).

The proofs of Theorem 7 and 8 are clear and so we omit them.

If every $A_{i m}, B_{k m}$ is an equilibrium, then hypotheses $\mathrm{H}_{4}, \mathrm{H}_{5}, \mathrm{H}_{7}$, and $\mathrm{H}_{8}$ can be easily checked, for example, by analysing the type of every equilibrium. The hypothesis $\mathrm{H}_{5}$ also can be checked by the Dulac Criterion. The hypothesis $\mathrm{H}_{7}$ shows that, for the two-dimensional subsystem which is formed by populations $x_{i}$ and $x_{j}$, it cannot occur that population $x_{i}$ persists and population $x_{j}$ extinguishes at $t \rightarrow+\infty$ and $t \rightarrow-\infty$ (there are $x, y \in R_{+}^{2}\left(x_{i}, x_{j}\right)$ such that $\left.\Lambda^{+}(x) \cup \Lambda^{-}(y) \subset A_{i}\right)$. The hypothesis $\mathrm{H}_{8}$ shows that, if all populations exist, then they cannot tend to the equilibrium stations $A_{i m}, B_{k m}$ on the boundary $R_{0}^{3}$ as $t \rightarrow+\infty$. The number $f_{i}(0,0,0)$ represents the intrinsic growth of population $x_{i}$, if $f_{i}(0,0,0)>0 \quad(<$ 0 ), then population $x_{i}$ can survive (must extinguish) in the absence of populations $x_{j}$ and $x_{k}$. Therefore hypotheses $\mathrm{H}_{9}-\mathrm{H}_{11}$ show that there is a population such that its intrinsic growth is positive.

For the two-predators and a common-prey model, the three-species food chains model, and the competitive model, the hypotheses and conditions of Theorem 7 or 8 can often be verified in order to obtain persistence and uniform persistence. For the two-preys and one-predator model, a theorem which is similar to Theorem 7 can often be checked in order to obtain persistence and uniform persistence. The works about this can be seen in $[6,7]$.

We have obtained necessary and sufficient conditions for system $F$ to be persistent and uniformly persistent under certain natural hypotheses. If any of these principal hypotheses are not satisfied, the system $F$ may not persist or uniformly persist. Examples may be found in the literatures [5, 10, 12, 13]. 


\section{REFERENCES}

[1] N. Bhatia and G. Szego, Dynamical Systems, Stability Theory and Applications, Lecture Notes in Math., No. 35, Springer-Verlag, New York, 1967

[2] G. Butler, H. Freedman, and P. Waltman, Uniformly persistent systems, Proc. Amer. Math. Soc. 96, 425-430 (1986)

[3] G. Butler and P. Waltman, Persistence in dynamical systems, J. Differential Equations 63, 255-263 (1986)

[4] A. Fonda, Uniformly persistent semidynamical systems, Proc. Amer. Math. Soc. 104. 111-116 (1988)

[5] H. Freedman, Deterministic Mathematical Models in Populations Ecology, Marcel Dekker, New York, 1980

[6] H. Freedman and P. Waltman, Persistence in models of three interacting predator-prey populations, Math. Biosci. 68, 213-231 (1984)

[7] H. Freedman and P. Waltman, Persistence in a model of three competitive populations, Math. Biosci. 73, 89-101 (1985)

[8] T. Gard, Persistence in food chains with general interactions, Math. Biosci. 51, 165-174 (1980)

[9] M. Hirsch and C. Pugh, Stable Manifolds and Hyperbolic Sets, Global Analysis, Proc. Symp. Pure Math., Vol. 14, Amer. Math. Soc., 1970

[10] S. Hsu, S. Hubbell, and P. Waltman, Competing predators, SIAM J. Appl. Math. 35, 617-625 (1978)

[11] V. Hutson and G. Vickers, A criterion for permanent coexistence of species, with an application to a two-prey one-predator system, Math. Biosci. 63, 253-269 (1983)

[12] R. May and W. Leonard, Nonlinear aspects of competition between three species, SIAM J. Appl. Math. 29, 243-253 (1975)

[13] P. Schuster, K. Sigmund, and R. Wolff, On w-limits for competition between three species, SIAM J. Appl. Math. 37, 49-54 (1979)

[14] S. Smale, Differentiable dynamical Systems, Bull. Amer. Math. Soc. 73, 747-817 (1967) 